\title{
Title: Attosecond Chronoscopy of Electron Scattering in Dielectric
}

\section{Nanoparticles}

Authors: L. Seiffert ${ }^{1}$, Q. Liu ${ }^{2,3}$, S. Zherebtsov ${ }^{2,3}$, A. Trabattoni ${ }^{4,5}$, P. Rupp ${ }^{2,3}$, M. C. Castrovilli ${ }^{6}$, M. Galli ${ }^{4,6}$, F. Süßmann ${ }^{2,3}$, K. Wintersperger ${ }^{2}$, J. Stierle ${ }^{2}$, G. Sansone ${ }^{4,6}$, L. Poletto ${ }^{7}$, F. Frassetto ${ }^{7}$, I. Halfpap ${ }^{8}$, V. Mondes ${ }^{8}$, C. $\mathrm{Graf}^{8}$, E. Rühl ${ }^{8}$, F. Krausz ${ }^{2,3}$, M. Nisoli ${ }^{4,6}$, T. Fennel ${ }^{1,9, *}$, F. Calegari $^{5,6,10, *}$, M. F. Kling ${ }^{2,3, *}$

\section{Affiliations:}

${ }^{1}$ Institute of Physics, University of Rostock, Albert-Einstein-Str. 23, D-18059 Rostock, Germany.

${ }^{2}$ Max Planck Institute of Quantum Optics, Hans-Kopfermann-Str. 1, D-85748 Garching, Germany

${ }^{3}$ Department of Physics, Ludwig-Maximilians-Universität München, Am Coulombwall 1, D85748 Garching, Germany

${ }^{4}$ Department of Physics, Politecnico di Milano, Piazza L. Da Vinci 32, I-20133 Milano, Italy

${ }^{5}$ Center for Free-Electron Laser Science, DESY, Notkestr. 85, 22607 Hamburg, Germany

${ }^{6}$ National Research Council of Italy, Institute for Photonics and Nanotechnologies, Piazza L. Da Vinci 32, I-20133 Milano, Italy

${ }^{7}$ National Research Council of Italy, Institute for Photonics and Nanotechnologies, via Trasea 7, I-35131 Padova, Italy

${ }^{8}$ Physical Chemistry, Freie Universität Berlin, Takustr. 3, D-14195 Berlin, Germany

${ }^{9}$ Max-Born-Institut, Max-Born-Straße 2A, 12489 Berlin, Germany

${ }^{10}$ Department of Physics, University of Hamburg, Jungiusstr. 9, 20355 Hamburg, Germany

*Correspondence to: Matthias F. Kling (matthias.kling@lmu.de), Francesca Calegari (francesca.calegari@desy.de), or Thomas Fennel (thomas.fennel@uni-rostock.de). 


\section{Introductory paragraph:}

Scattering of electrons in dielectric materials is at the heart of laser nanomachining ${ }^{1}$, light-driven

electronics ${ }^{2}$, and radiation damage ${ }^{3-5}$. Here, we demonstrate real-time access to electron scattering by implementing attosecond streaking spectroscopy on dielectric nanoparticles: photoelectrons are generated inside the nanoparticles and both their transport through the material and photoemission are tracked on an attosecond time scale. We develop the framework for attosecond streaking spectroscopy in dielectrics and identify that the presence of the internal field inside the material cancels the influence of elastic scattering, enabling the selective characterization of the inelastic scattering time. The approach is demonstrated on silica nanoparticles, where an inelastic mean free path is extracted for $20-30 \mathrm{eV}$. The new vistas into electron transport can be obtained for a broad range of energies and dielectric solids and liquids including water, which can be studied in the form of droplets. 


\section{Main Text:}

The interaction of a dielectric material with photons that have above band-gap energies inevitably leads to the production of hot electrons propagating through the material. During propagation these electrons can undergo a number of elastic and inelastic scattering events. The inelastic scattering of such electrons can give rise to rapid carrier multiplication via impact ionization, enabling laser nanomachining ${ }^{1}$ and limiting the scaling of electronic signal processing towards optical (PHz-scale) frequencies using extremely strong electric fields ${ }^{2}$ by dielectric breakdown. Moreover, secondary electrons represent the main source of radiation damage following tissue irradiation. Thus knowledge of inelastic electron scattering in a dielectric, such as liquid water, is essential to fully understand how radiation affects living organisms ${ }^{3-5}$.

To understand the phenomena above, we must be able to study collision dynamics in dielectrics for energies ranging from the band-gap energy up to the hard-X-ray regime. However, methods for measuring collision dynamics have been mostly limited to electron energies above $\approx 50 \mathrm{eV}^{6}$. These methods include the measurement of inelastic mean free paths (IMFPs), where a mean free path is the average distance that an electron will travel in a solid between adjacent collision events. Standard IMFP measurements are based on the dielectric description of inelastic scattering of charged particles in condensed matter ${ }^{7}$ and elastic peak electron spectroscopy. While progress has been made toward determining IMFPs for very low kinetic energy (of a few eV) by angle-resolved photoelectron spectroscopy ${ }^{8}$, data in the tens of $\mathrm{eV}$ range, where the impact ionization of valenceband electrons strongly dominates the IMFP, is largely missing. Moreover, amorphous dielectric solids such as $\mathrm{SiO}_{2}$ and liquid water, which can be accessed with our approach, are challenging for rigorous ab-initio calculations of IMFPs. 
For electron energies in the tens-of-eV range, collisions typically occur in the sub-femtosecond range. We have access to this timescale via attosecond streaking 9 : photoemission inside a solid is initiated by an extreme ultraviolet (XUV) pulse, and the momentum change of released electrons due to a second near-infrared (NIR) pulse is measured as a function of XUV/NIR pulse delay (see methods). To date, this metrology has only been applied to metals ${ }^{10-19}$ or adlayer-covered metals $^{20,21}$, where the NIR streaking field only takes effect on the surface of the material. The physics for dielectrics, however, is fundamentally different as the released electrons are streaked by the NIR field also inside the solid. Most importantly, until now accumulative charging induced by the XUV pulse has prevented the application of attosecond streaking to dielectrics. Here, we solve this problem by using a continuous stream of nanoscopic targets. Our proof-of-concept study was focused on silicon dioxide, the most important dielectric material in microelectronics and optics.

In the experiment depicted in Fig. 1, a stream of isolated 50-nm, $\mathrm{SiO}_{2}$ nanospheres was delivered into the laser interaction region by aerodynamic lens focusing ${ }^{22-26}$. Emitted electrons were detected by a kilohertz, single-shot velocity-map imaging (VMI) spectrometer. This setup can differentiate images that contain only reference gas from those with nanoparticles, as reference-gas-only frames contain significantly fewer electrons than do those containing nanoparticles, see Fig. 2(a). Further frame-selection was obtained by taking into account the asymmetric momentum distribution of nanoparticle electrons originating from shadowing ${ }^{22,25,27}$ of the XUV field, thus providing an efficient sorting method, see Fig. 2(b) and (c). This data discrimination provided us with nanoparticle and reference streaking spectrograms (such as those 
in Fig. 3) from the very same experimental data set, i.e. for identical laser conditions from a single pulse delay scan. Figures 2(d) and (e) show discriminated momentum data, averaged over millions of shots, for the reference medium and nanoparticles, respectively.

When analyzed as a function of the delay of the NIR with respect to the XUV pulses, $\Delta t$, electron kinetic energy spectra derived from the projected momentum form streaking spectrograms with pronounced delay-dependent oscillations for gas and nanoparticles, see Figs. 3(a) and (b). The gas reference essentially reflects the NIR pulse vector potential. It further includes temporal shifts due to the Wigner delay ${ }^{28}$ and effects from Coulomb-laser coupling ${ }^{29,30}$. As the long-range potentials of gas and nanoparticles are both Coulomb-like, these shifts are assumed comparable for gas and nanoparticles. They effectively cancel each other in the relative delay between the spectrograms, up to remaining short-range effects that are assumed small away from resonances ${ }^{31}$. To extract the delays, we consider isocontour lines for different asymptotic energies (e.g. blue and red dotted lines for $25 \mathrm{eV}$ ). Individual energy-dependent streaking delays are determined from fits to the respective filtered contour lines (see SI). The streaking delays $\delta t_{S i O_{2}}$ for $\mathrm{SiO}_{2}$ and $\delta t_{\text {gas }}$ for the reference gas (symbols in Fig. 3(d)) exhibit similar energy dependent tilts, which are attributed to the XUV pulse properties (XUV chirp, see SI for details). Measured nanoparticle frames still contain a small contribution from residual gas, see Fig. 3(e).

We simulated electron emission from nanoparticles by trajectory-based Mean-field Mie MonteCarlo transport calculations $\left(\mathrm{M}^{3} \mathrm{C}\right)^{32}$. XUV photoelectrons generated inside the material are propagated in the NIR near-field of the particle and experience elastic and inelastic collisions which have energy-dependent cross-sections (see SI for details). The energy-dependent streaking 
delays, $\delta t_{\mathrm{SiO}_{2}}$, extracted from simulated streaking spectrograms for pure nanoparticles (Fig. 3(c)) exhibit a tilt similar to that of the experiments (black line in Fig. 3(d)) and a global relative streaking delay $\delta t_{r e l}=\delta t_{S i_{2}}-\delta t_{\text {gas }}$ with respect to the delay, $\delta t_{\text {gas }}$, calculated for gas. The gas data (blue symbols) can be fully reproduced (blue line) by taking into account the XUV chirp.

For a quantitative comparison, we combined simulated spectrograms of gas and nanoparticles using a single mixing factor such that the signal ratio found in the experiments is reproduced, see Fig. 3(e). Streaking delays extracted from this mixed nanoparticle data (red line in Fig. 3(d)) show good agreement with the experiment. This supports that the model accurately reflects the relevant phenomena under scrutiny and that other factors are of minor importance.

The agreement between the experimental data and simulation results motivates a closer analysis of contributions to the relative streaking delay in order to gain insight into the relevant processes. The first two contributions to the absolute $\mathrm{SiO}_{2}$ streaking delay $\delta t_{\mathrm{SiO}_{2}}=\delta t_{\text {offset }}+\delta t_{\text {chirp }}+$ $\delta t_{\text {fields }}+\delta t_{\text {coll }}$ describe the carrier-envelope phase of the streaking field plus the XUV-NIR delay axis offset $\left(\delta t_{\text {offset }}\right)$ and the XUV chirp ( $\left.\delta t_{\text {chirp }}\right)$. These two terms represent the residual gas delay and thus cancel out in the resulting relative streaking delay $\delta t_{\text {rel }}=\delta t_{\mathrm{SiO}_{2}}-\delta t_{\mathrm{gas}}=$ $\delta t_{\text {fields }}+\delta t_{\text {coll }}$ (see SI for details). Since the contribution $\delta t_{\text {fields }}$, characterizing the retardation and inhomogeneity effects of the NIR and XUV near-fields, is negligible (see SI), the remaining term from electron scattering within the solid, $\delta t_{\text {coll }}$, provides the dominant contribution that is illustrated in Fig. 4. In the relevant energy range, interband excitations constitute the dominant inelastic scattering channel ${ }^{33}$ and result in an energy loss comparable to the band gap energy $(\approx$ $9 \mathrm{eV}$ for $\mathrm{SiO}_{2}$ ), removing the inelastically scattered electrons from the spectral region of interest, 
i.e. the high energy part of the streaking trace. We therefore focus on electrons without inelastic collisions, which determine the high-energy signals, see inset of Fig. 3(d). For clarity, we discuss the effect for a fully transparent material (unit permittivity). Electrons with charge $q$ generated at time $t_{0}$ with initial momentum $\mathrm{p}_{0}$ have a final momentum $\mathrm{p}_{\mathrm{f}}=\mathrm{p}_{0}+\mathrm{qA}\left(\mathrm{t}_{0}\right)$ when escaping along the surface normal (green arrow in Fig. 4(a)) without any collisions. The streaking trace that results from these directly emitted electrons (green curve in Fig. 4(b)) characterizes the streaking field vector potential, $\mathrm{A}(\mathrm{t})$, and is thus equivalent to gas-only traces.

In contrast, electrons that leave after elastic, isotropic scattering at time $t_{c}$ (red symbol in Fig. 4(a)) are essentially indistinguishable from direct electrons generated at time $t_{c}$ (see SI for details). Each elastic collision acts as a new "birth" for an electron such that electrons with final elastic scattering time $t_{\mathrm{c}}$ have an average final momentum $\left\langle\mathrm{p}_{\mathrm{f}}\right\rangle \approx \mathrm{p}_{0}+\mathrm{qA}\left(\mathrm{t}_{\mathrm{c}}\right)$, yielding a streaking trace shifted by $t_{\mathrm{c}}$ (red curve in Fig. 4(b)). The final spectrogram is an average of shifted streaking traces, each weighted with the corresponding emission current. As the latter scales with the population decay resulting from interband excitations, the streaking delay is very sensitive to the inelastic scattering time (Fig. 4(c)).

$\mathrm{M}^{3} \mathrm{C}$ simulations with varying scattering parameters reveal the quantitative effects of elastic and inelastic scattering on the relative streaking delays (Fig. 5(a)), namely a weak dependence on the elastic scattering time, and a strong dependence on the inelastic scattering time. The impact of a material's permittivity at the streaking field wavelength is shown in Fig. 5(b). Irrespective of the permittivity, represented by the field attenuation factor $\alpha=1 / \varepsilon_{r}$, the streaking delay increases with inelastic scattering time. However, in both the transparent $(\alpha \rightarrow 1)$ and the metal $(\alpha \rightarrow 0)$ 
limits, the delays remain strongly influenced by the elastic scattering time. With increasing elastic scattering time, delays decrease toward the transparent limit but increase toward the metallic limit. These opposing trends reflect the effect of the internal field attenuation and, most importantly, almost vanish for $\alpha \approx 0.3$. The identification of this cancelation is one of the major results of our analysis as it enables the direct retrieval of inelastic scattering times for a range of dielectric materials. The streaking delay's high sensitivity to inelastic collision time justifies its extraction from the full $\mathrm{M}^{3} \mathrm{C}$ simulations, which match the experimental data in Fig. 3(d) using a scaled Lotz cross section. We find $\tau_{\text {inel }} \sim 400$ as at $25 \mathrm{eV}$ in $\mathrm{SiO}_{2}$. Figure 5(c) shows comparison of the resulting electron IMFP in $\mathrm{SiO}_{2}$ obtained here between 20 and $30 \mathrm{eV}$ with results found in the literature ${ }^{6,34-}$ ${ }^{36}$. Note that best agreement is found with the work of Tanuma et al., who state that their data in the range of $10-40 \mathrm{eV}$ is unreliable and only serves as a trend ${ }^{34}$. With IMFP obtained via the demonstrated approach the predictive capability of theoretical models for scattering in dielectrics can now be thoroughly tested.

In summary, our joint experimental and theoretical work uncovers the physics encoded in attosecond streaking on dielectrics and resolves the characterization problem of inelastic scattering in these materials. Attosecond streaking in dielectrics differs from metals where the NIR streaking field only takes effect on the surface of the material. The presence of the field inside the dielectric determines the importance of elastic and inelastic scattering on the streaking delay. We found that for most dielectric materials the streaking delay is largely independent of the elastic scattering time thus enabling the characterization of inelastic scattering. The accessible energy range only depends on the photon energy of the attosecond pulse, which even currently covers tens of $\mathrm{eV}$ to $\mathrm{keV}$ and might be further extended in the future. Our approach permits characterization of inelastic scattering in a wide range of dielectric solids and liquids, including amorphous dielectrics such as 
water, which could be inserted in the form of droplets or as a thin jet. Direct access to inelastic mean-free path in dielectrics for a wide range of energies including the otherwise inaccessible range below $50 \mathrm{eV}$ holds promise for improving our understanding of radiation damage.

\section{Acknowledgments:}

We are grateful for support by the EU via the ERC grants ATTOCO (no. 307203), STARLIGHT (no. 637756), and ELYCHE (no. 227355), LASERLAB-EUROPE (no. 284464, EU Seventh Framework Programme), the Max Planck Society and the DFG through the Cluster of Excellence: Munich Centre for Advanced Photonics (MAP), SPP1391, SPP1840, and SFB 652/3. We acknowledge the computing time provided by the North-German super-computing center HLRN (project ID mvp00011). We acknowledge help in editing the manuscript from B. Steffl and fruitful discussions with V. S. Yakovlev, H. J. Wörner, and R. Signorell.

\section{Author contributions:}

L.S., Q.L., S.Z., and A.T. contributed equally to this work. M.F.K., F.C., and T.F. conceived the measurement concept and implementation. Q.L., S.Z., A.T., M.C.C., M.G., P.R., and F.C. performed the measurements. F.S., K.W., J.S., G.S., L.P., and F.F. were involved in the setup of the experiment and experimental infrastructure. I.H., V.M., C.G., and E.R. prepared and characterized the $\mathrm{SiO}_{2}$ nanoparticles. L.S. and T.F. developed the simulation model and performed the simulations. L.S., Q.L., S.Z., A.T., T.F., F.C., and M.F.K. evaluated, analyzed and interpreted the results. All authors discussed the results and contributed to the final manuscript. 


\section{Additional information:}

Supplementary information is available in the online version of the paper.

\section{Competing financial interests:}

None

\section{Data availability:}

The data that support the plots within this paper and other findings of this study are available from the corresponding author upon reasonable request.

\section{References:}

1 Balling, P. \& Schou, J. Femtosecond-laser ablation dynamics of dielectrics: basics and applications for thin films. Rep. Prog. Phys. 76, 036502 (2013).

2 Krausz, F. \& Stockman, M. I. Attosecond metrology: from electron capture to future signal processing. Nat. Photon. 8, 205-213 (2014).

3 Toburen, L. H. et al. Electron Emission from Amorphous Solid Water Induced by Passage of Energetic Protons and Fluorine Ions. Rad. Res. 174, 107-118 (2010).

4 Garrett, B. C. et al. Role of Water in Electron-Initiated Processes and Radical Chemistry: Issues and Scientific Advances. Chem. Rev. 105, 355-390 (2005).

5 Caleman, C. et al. Radiation damage in biological material: Electronic properties and electron impact ionization in urea. Europhys. Lett. 85, 18005 (2009).

6 Siddharth, H. P., Vaishnav, B. G. \& Joshipura, K. N. Electron inelastic mean free paths in solids: A theoretical approach. Chin. Phys. B 21, 093402 (2012).

7 Powell, C. J. Inelastic scattering of kilovolt electrons by solids and liquids: determination of energy losses, cross sections, and correlations with optical data. Health Phys. 13, 1265-1276 (1967).

8 Goldmann, M., Miguel-Sánchez, J., West, A. H. C., Yoder, B. L. \& Signorell, R. Electron mean free path from angle-dependent photoelectron spectroscopy of aerosol particles. $J$. Chem. Phys. 142, 224304 (2015).

9 Itatani, J. et al. Attosecond streak camera. Phys. Rev. Lett. 88, 173903 (2002).

10 Cavalieri, A. L. et al. Attosecond spectroscopy in condensed matter. Nature 449, 10291032 (2007).

11 Neppl, S. et al. Attosecond time-resolved photoemission from core and valence states of magnesium. Phys. Rev. Lett. 109, 087401 (2012). 
12 Okell, W. A. et al. Temporal broadening of attosecond photoelectron wavepackets from solid surfaces. Optica 2, 383-387 (2015).

13 Locher, R. et al. Energy-dependent photoemission delays from noble metal surfaces by attosecond interferometry. Optica 2, 405-410 (2015).

14 Borisov, A. G., Sánchez-Portal, D., Kazansky, A. K. \& Echenique, P. M. Resonant and nonresonant processes in attosecond streaking from metals. Phys. Rev. B 87, 121110 (2013).

15 Zhang, C.-H. \& Thumm, U. Attosecond photoelectron spectroscopy of metal surfaces. Phys. Rev. Lett. 102, 123601 (2009).

16 Kelkensberg, F., Koenderink, A. F. \& Vrakking, M. J. J. Attosecond streaking in a nanoplasmonic field. New J. Phys. 14, 093034 (2012).

17 Li, J., Saydanzad, E. \& Thumm, U. Retrieving plasmonic near-field information: A quantum-mechanical model for streaking photoelectron spectroscopy of gold nanospheres. Phys. Rev. A 94, 051401 (2016).

18 Prell, J. S., Borja, L. J., Neumark, D. M. \& Leone, S. R. Simulation of attosecondresolved imaging of the plasmon electric field in metallic nanoparticles. Ann. Phys. 525, 151-161 (2013).

19 Süßmann, F. \& Kling, M. F. Attosecond nanoplasmonic streaking of localized fields near metal nanospheres. Phys. Rev. B 84, 121406(R) (2011).

20 Neppl, S. et al. Direct observation of electron propagation and dielectric screening on the atomic length scale. Nature 517, 342-346 (2015).

21 Liao, Q. \& Thumm, U. Attosecond time-resolved streaked photoemission from Mgcovered W(110) surfaces. Phys. Rev. A 92, 031401 (2015).

22 Wilson, K. R. et al. Size-Dependent Angular Distributions of Low-Energy Photoelectrons Emitted from $\mathrm{NaCl}$ Nanoparticles. Nano Lett. 7, 2014-2019 (2007).

23 Ellis, J. L. et al. Materials Properties and Solvated Electron Dynamics of Isolated Nanoparticles and Nanodroplets Probed with Ultrafast Extreme Ultraviolet Beams. $J$. Phys. Chem. Lett. 7, 609-615 (2016).

24 Hickstein, D. D. et al. Observation and Control of Shock Waves in Individual Nanoplasmas. Phys. Rev. Lett. 112, 115004 (2014).

25 Süßmann, F. et al. Field propagation-induced directionality of carrier-envelope phasecontrolled photoemission from nanospheres. Nat. Commun. 6, 7944 (2015).

26 Zherebtsov, S. et al. Controlled near-field enhanced electron acceleration from dielectric nanospheres with intense few-cycle laser fields. Nat. Phys. 7, 656 (2011).

27 Signorell, R. et al. Nanofocusing, shadowing, and electron mean free path in the photoemission from aerosol droplets. Chem. Phys. Lett. 658, 1-6 (2016).

28 Wigner, E. P. Lower Limit for the Energy Derivative of the Scattering Phase Shift. Phys. Rev. 98, 145-147 (1955).

29 Smirnova, O., Mouritzen, A. S., Patchkovskii, S. \& Ivanov, M. Y. Coulomb-laser coupling in laser-assisted photoionization and molecular tomography. Journal of Physics B: Atomic, Molecular and Optical Physics 40, F197 (2007).

30 Zhang, C. H. \& Thumm, U. Electron-ion interaction effects in attosecond time-resolved photoelectron spectra. Phys. Rev. A 82, 043405 (2010).

31 Dahlström, J. M. et al. Theory of attosecond delays in laser-assisted photoionization. Chemical Physics 414, 53-64 (2013). 
32 Seiffert, L. et al. Competition of single and double rescattering in the strong-field photoemission from dielectric nanospheres. Appl. Phys. B 122, 1-9 (2016).

33 Kuhr, J. C. \& Fitting, H. J. Monte Carlo simulation of electron emission from solids. J. Electr. Spec. \& Rel. Phen. 105, 257-273 (1999).

34 Tanuma, S., Powell, C. J. \& Penn, D. R. Calculations of electron inelastic mean free paths. III. Data for 15 inorganic compounds over the $50-2000 \mathrm{eV}$ range. Surf. \& Interf. Anal. 17, 927-939 (1991).

35 Ashley, J. C. \& Anderson, V. E. Interaction of low-energy electrons with silicon dioxide. J. El. Spectr. \& Rel. Phen. 24, 127-148 (1981).

36 Akkerman, A. et al. Inelastic Electron Interactions in the Energy Range $50 \mathrm{eV}$ to $10 \mathrm{keV}$ in Insulators: Alkali Halides and Metal Oxides. Phys. Stat. Solidi (b) 198, 769-784 (1996). 


\section{Figures:}

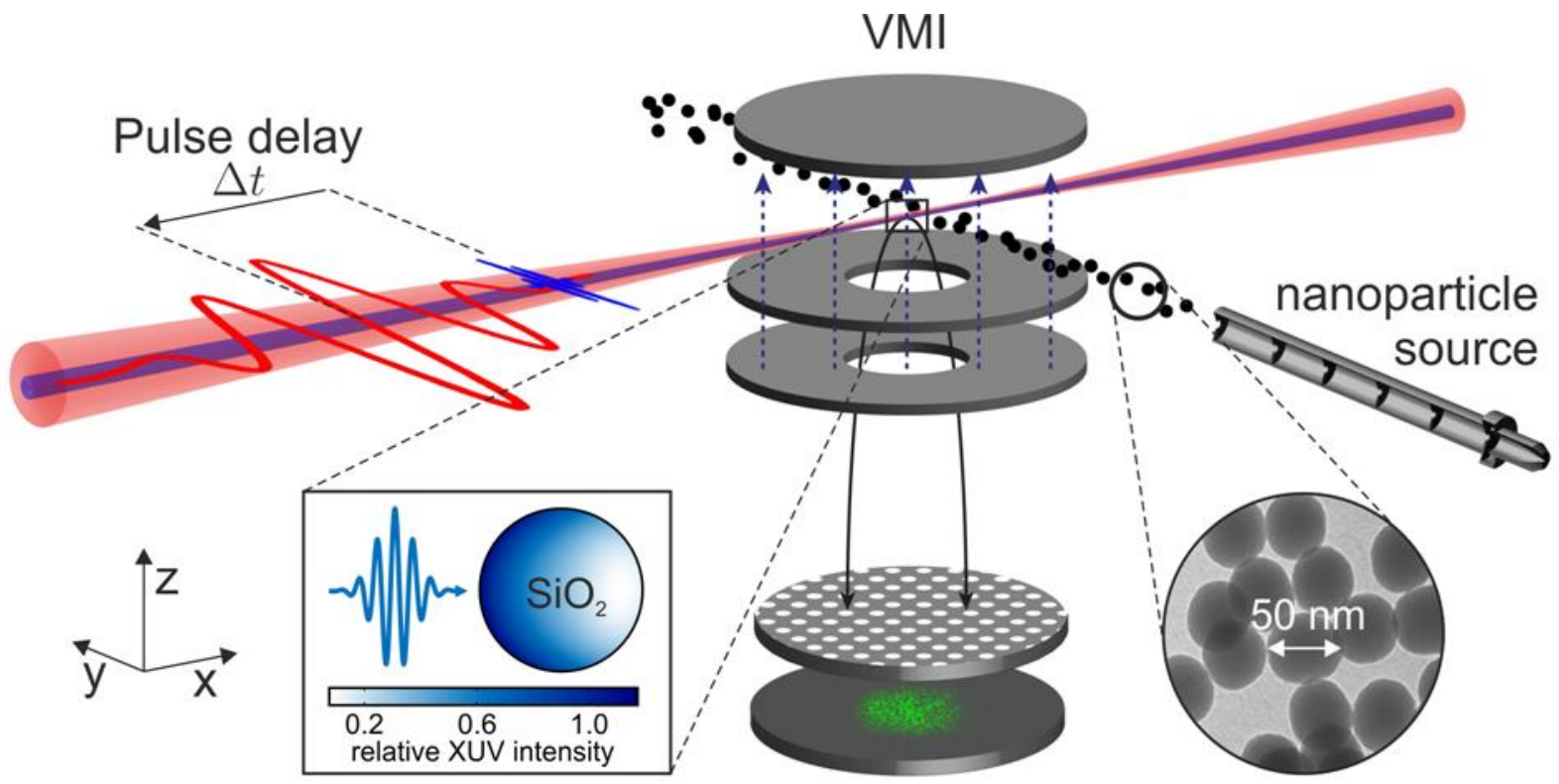

Figure 1: Schematic setup of the attosecond streaking experiment. A beam of isolated nanoparticles is hit by isolated attosecond XUV pulses and synchronized few-cycle NIR pulses. Emitted electrons are recorded with a VMI spectrometer. Right inset: Transmission-electron-microscopy image of the $\mathrm{SiO}_{2}$ nanoparticles. Left inset: Mie calculation of the XUV attenuation (shadowing). 

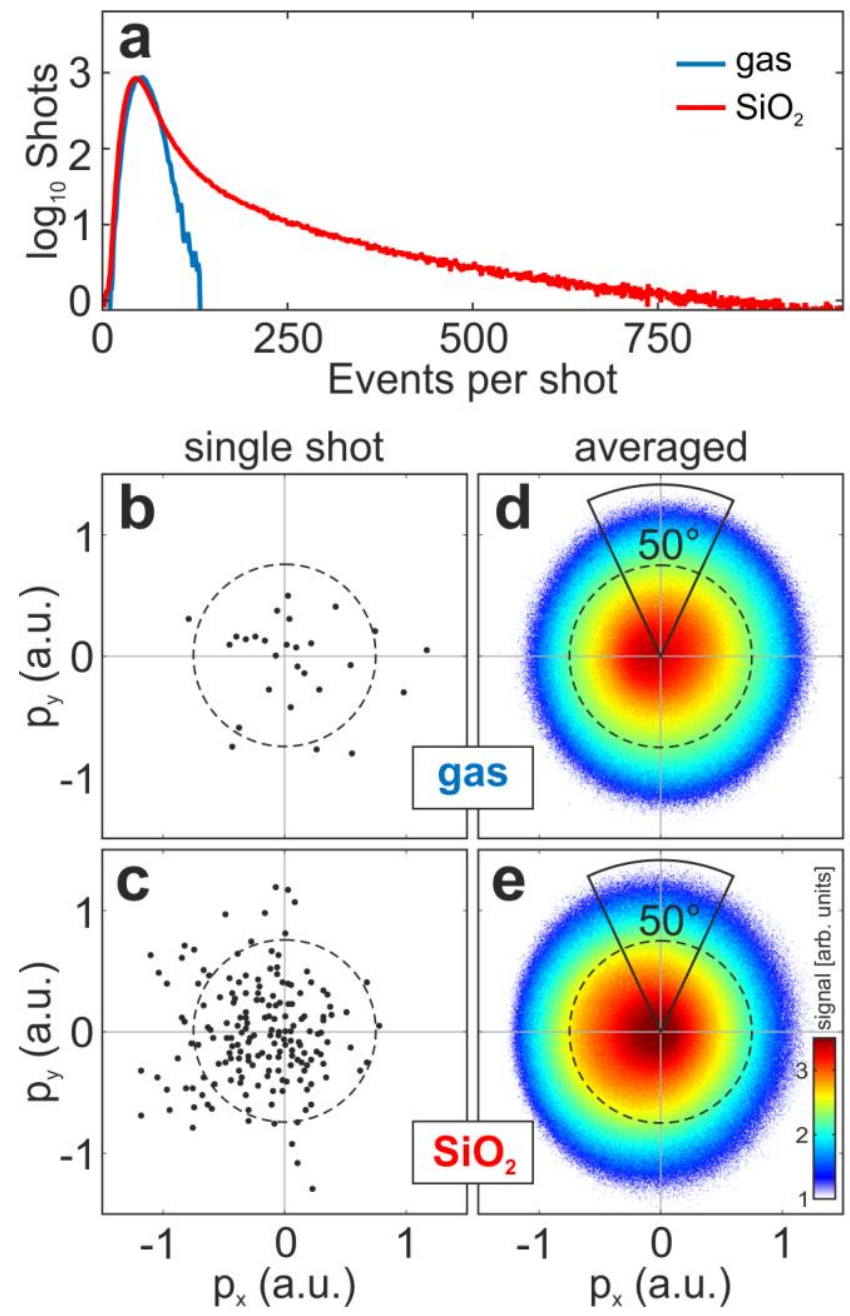

Figure 2: Discrimination of nanoparticle and gas data (a) Histogram of the events per shot measured for residual gas only and with $\mathrm{SiO}_{2}$ nanoparticles (as indicated). (b,c) Typical single shot images containing contributions from the residual gas only (b) and nanoparticles (c) measured for zero delay. Photoelectrons emitted from nanoparticles exhibit asymmetric momentum distributions that originate from XUV shadowing. (d,e) Respective averaged momentum distributions measured for zero delay. For low momenta ( $<0.75$ a. u., inside the dashed circles) the signal contains additional background contribution (see SI). 

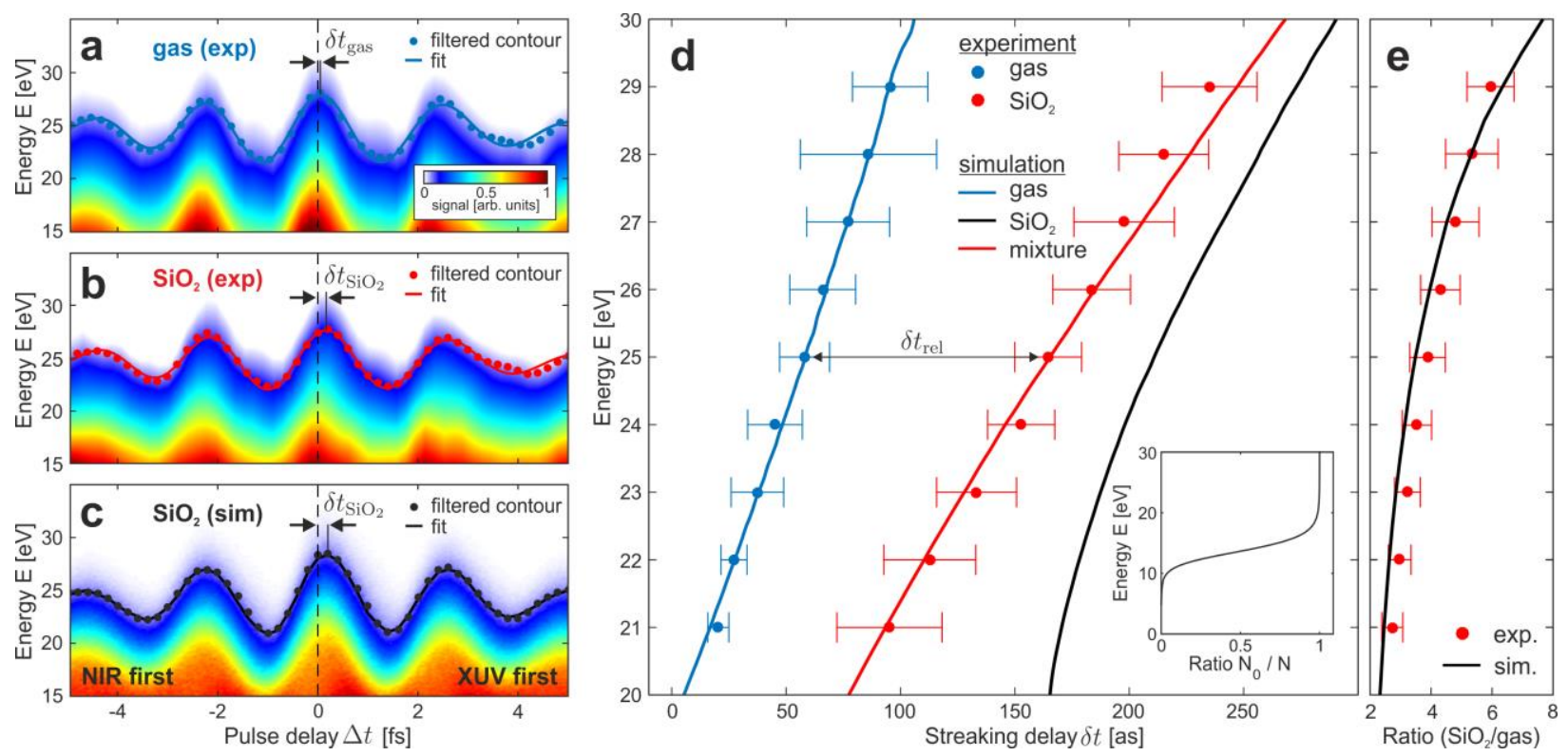

Figure 3: Streaking delay analysis. (a-c) Attosecond spectrograms measured for residual gas and nanoparticles and as predicted by $\mathrm{M}^{3} \mathrm{C}$ simulations (as indicated) obtained from angular integration of projected momentum maps over $\pm 25^{\circ}$ around the laser polarization direction (see Fig. 2(d,e) and SI for details). For the relative delay analysis energy-dependent frequency-filtered isolines were fitted with fewcycle waveforms, whose carrier phases define the streaking delay $\delta t$ (cf. symbols and curves in (a-c), see SI for details). (d) Energy-dependent streaking delays measured for residual gas (blue symbols) and nanoparticles (red symbols). The data represents an average over three scans performed under similar conditions (error bars indicate the deviation of the individual data sets). Solid lines show corresponding simulation results for gas (blue), nanoparticles (black) and their mixture (red) using an XUV chirp of $-7 \times 10^{-3} \mathrm{fs}^{2}$. Inset: Relative contribution of electrons without inelastic collisions in the $\mathrm{M}^{3} \mathrm{C}$-simulations. (e) Measured energy-dependent ratio of nanoparticle signal relative to residual gas (red) and result obtained from combining simulated gas and nanoparticle spectrograms (black). 

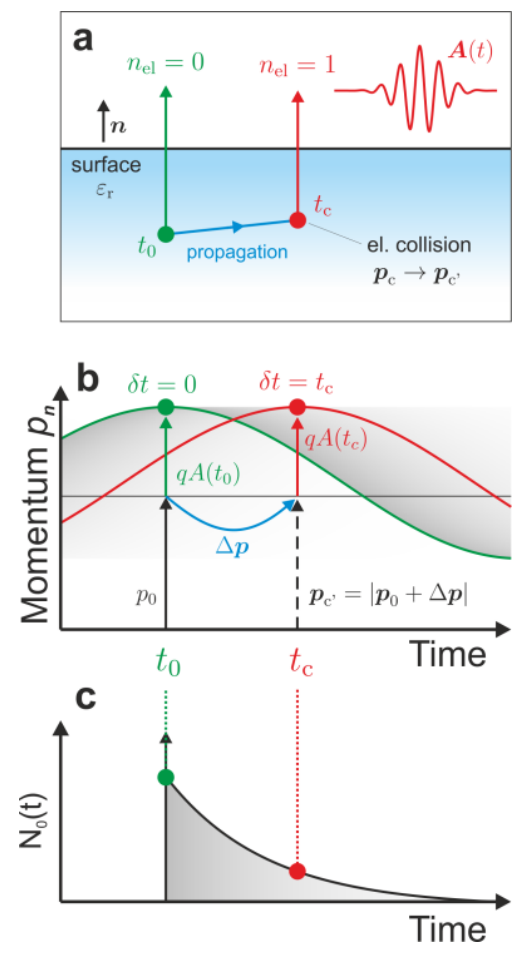

Figure 4: Impact of elastic and inelastic scattering on the streaking delays. (a) Photoemission of electrons generated at time $t_{0}$ with initial momentum $\boldsymbol{p}_{\mathbf{0}}$ from an ideal transparent material (unit permittivity) without collisions (green) and following an elastic collision (red) at time $t_{\mathrm{c}}$ after propagation inside the material (blue arrow). (b) The final momentum $\boldsymbol{p}_{\boldsymbol{f}}=\left|\boldsymbol{p}_{\mathbf{0}}\right| \boldsymbol{n}+q \boldsymbol{A}\left(t_{0}\right)$ for unscattered electrons with charge $q$ emitted parallel to the surface normal $\boldsymbol{n}$ reflects the NIR pulse vector potential $\boldsymbol{A}(t)=$ $A_{0} \boldsymbol{n} \cos (\omega[t-\Delta t])$, resulting in a streaking trace with delay $\delta t=0$ (green curve). Elastically scattered electrons gain a momentum $\Delta \boldsymbol{p}=q\left[\boldsymbol{A}\left(t_{0}\right)-\boldsymbol{A}\left(t_{c}\right)\right]$ during propagation in the lab frame before the ingoing collision momentum $\boldsymbol{p}_{\mathbf{c}}=\boldsymbol{p}_{\mathbf{0}}+\Delta \boldsymbol{p}$ is directionally randomized. The final momentum for normal emission becomes $\boldsymbol{p}_{\boldsymbol{f}}=\left|\boldsymbol{p}_{\mathbf{c}}\right| \boldsymbol{n}+q \boldsymbol{A}\left(t_{c}\right)$. For an electron ensemble with isotropic initial momenta $\boldsymbol{p}_{\mathbf{0}}$, the shift $\Delta \boldsymbol{p}$ is distributed isotropically and thus roughly cancels on average in the initial momentum frame (see SI) such that $\left\langle p_{\mathrm{c}}\right\rangle \approx p_{0}$ for $\Delta p \ll p_{0}$. The average final momentum for normal emission is $\left\langle p_{f}\right\rangle \approx p_{0}+q A\left(t_{c}\right)$. The mean streaking trace is essentially shifted by $\delta t \approx t_{\mathrm{c}}$ compared to the direct emission (red vs. green curve). (c) Population of electrons without inelastic collisions leading to time-shifted contributions to the final streaking spectrogram. 

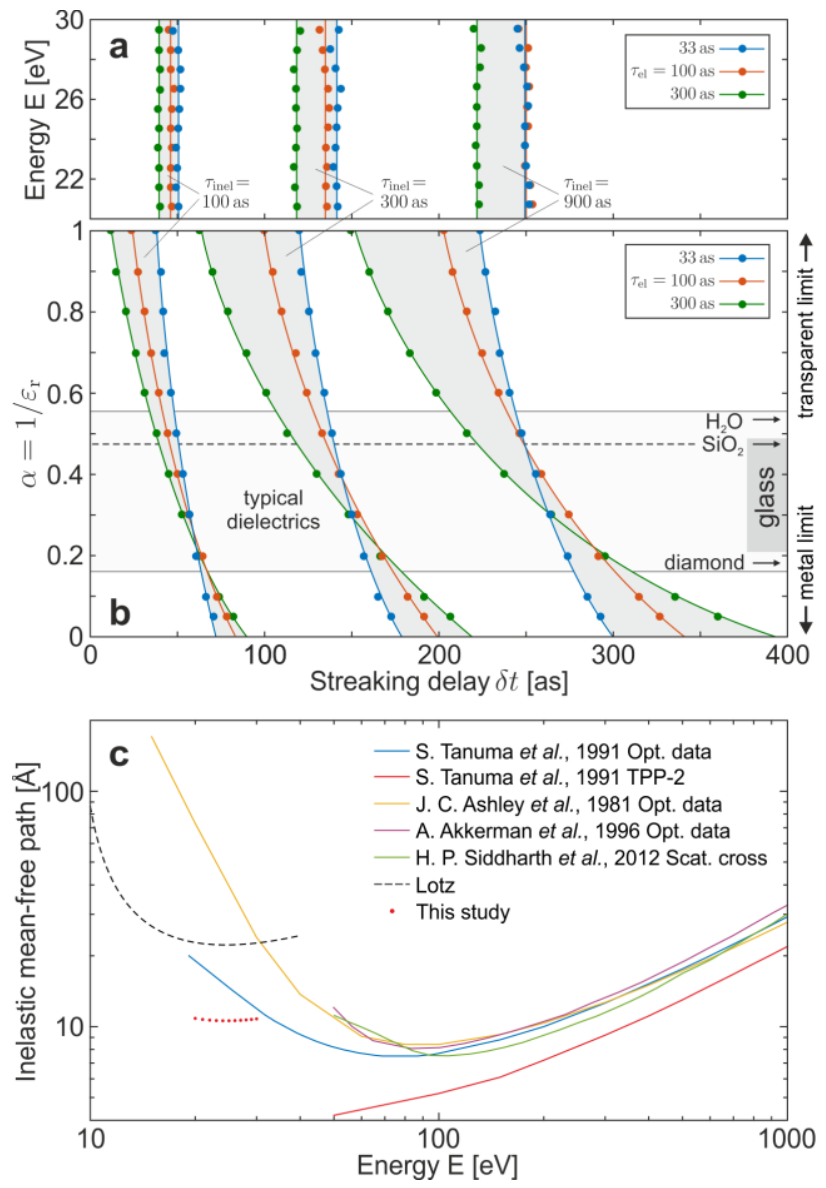

Figure 5: Extraction of the inelastic scattering time. (a) Streaking delays for $50 \mathrm{~nm} \mathrm{SiO}_{2}$ nanoparticles calculated from $\mathrm{M}^{3} \mathrm{C}$ for varying elastic and inelastic scattering times (as indicated). For clarity, we considered unchirped XUV pulses, non-dispersive material, and energy-independent inelastic and elastic scattering times, $\tau_{\text {inel }}$ and $\tau_{\text {el }}$, resulting in essentially energy-independent streaking delays. (b) The streaking delays as a function of the solids' permittivity at $720 \mathrm{~nm}$, expressed by the field attenuation factor $\alpha=$ $1 / \varepsilon_{r}$. Gray areas in (a) and (b) indicate the variation of the delays with the elastic scattering time. Arrows and the shaded rectangle in (b) indicate the range of $\alpha$ parameters for typical dielectrics; the dashed horizontal line marks the value for $\mathrm{SiO}_{2}$. (c) Energy dependent IMFPs obtained in previous work ${ }^{6,34-36}$ (solid lines, see SI for details). IMFP predicted by a simplified Lotz formula (dashed line). Note that the Lotz data is not shown for higher energies as the simplified model neglects lower lying bands. IMFPs from quantitative simulations of our experimental data using a scaled Lotz formula for the energy range 20-30 $\mathrm{eV}$ (red dots). 